\title{
Modeling of micrometre-sized molten metallic droplet impact on a solid wall
}

\author{
A. T. T. Tran \& M. M. Hyland \\ Department of Chemical and Materials Engineering, \\ University of Auckland, New Zealand
}

\begin{abstract}
Thermal spray coatings have been widely applied to enhance the appearance, adhesion, wear, oxidation, and corrosion resistance of materials in various industrial applications. Coatings form by the impact and spreading of high velocity molten coating droplets (splats) on a substrate. Coating buildup occurs as subsequent droplets overlap. A fundamental understanding of the complex interaction between a droplet and solid substrate can help to predict and improve the splat spreading process and thus the quality of coatings at a larger scale. In this study, a three dimensional model has been developed to simulate the impact, spreading and solidifying process of a micrometre-sized molten nickel droplet on a smooth stainless steel surface. The model is based on computational fluid dynamics (CFD), and combines a multiphase Eulerian fixed-grid model with a volume of fluid method (VOF) to track the droplet free surface. The effects of thermal contact resistance and contact angle on droplet shape were investigated. We demonstrate that high values of thermal contact resistance or contact angles can induce droplet breakup. The numerical results will provide new insights into the interpretation of empirical results as well as enhance the physical understanding of the fluid mechanism that leads to splashing

Keywords: CFD, contact angle, droplet deformation, droplet modelling, metallic droplet, solidification, splashing, VOF.
\end{abstract}

\section{Introduction}

Numerous challenges affect the various aspects of materials development, including the bonding of dissimilar materials and the design of effective multimaterial interfaces. These aspects play a major role in coatings, ink jet printing, 
metal matrix composites, chemical processes and coating deposition. Thermal spray coating, a technique that deposits particles of a coating material on substrates, has been widely applied to enhance the appearance, adhesion, wear, oxidation, surface fatigue, scratch and corrosion resistance of materials in various industries; such as aeronautics, automotive, medical, marine and electronics, to name a few. Coatings form as molten coating droplets with a high kinetic energy, which flatten out to form overlapping splats upon impact, solidifying and then bonding to the substrate. Thus, the performance of the coatings is closely linked with individual splat formation [1].

Many researchers have studied the splat-substrate interaction to predict and improve the spreading process and thus the quality of coatings at larger scales. However, the splashing problem is far from being fully understood, because the sequence of impact $\rightarrow$ spreading $\rightarrow$ solidification $\rightarrow$ breakup of the micrometrescale droplet occurs within microseconds and thus is too difficult for direct observation $[1,2]$. To overcome this limitation, numerical models have been extensively used to achieve better insights into droplet deformation $[3,4]$. There are a large number of numerical models of molten droplet impact on a solid surface. Since the first two-dimensional numerical model of a liquid drop splashing onto a flat surface was developed in 1967 by Harlow and Shannon [5], full three-dimensional models have been widely employed to examine splat formation and morphology in thermal spraying in recent [3,4]. In thermal spray coating, splat morphology is strongly dependent on thermal contact resistance and contact angle. Measurements of contact angle during droplet deformation process are necessary to accurately describe the fluid flow in particular at the contact line where the solid-liquid-gas come into effects. However, due to the droplet spreading process in thermal spray coating occurs within micro seconds that no experimental measurements of contact angle variation during droplet impact are available in the literature. In addition, the particle is at very velocity at impact, thus inertial force dominates the droplet deformation initially rather than the surface tension or viscosity of the droplet. Thermal contact resistance accounts for discontinuity at the interface between splat and substrate $[3,4,6]$. It was found that thermal contact resistance is a complex function of variety parameters: substrate surface conditions, spraying conditions, and the extent of contact at the substrate-splat interface. In addition, thermal contact resistance varies with time and position across the interface. Thus, the measurement of the real value of thermal contact resistance at each contact point for a micro-sized droplet is impossible. Alternatively, thermal contact resistance has been estimated based on the contact area between the splat and the substrate, using a one-dimensional analytical conduction model $[7,8]$. To reduce complexity, a constant value of thermal contact resistance is commonly used in numerical modeling of splat spreading and solidification process. Reported values of thermal contact resistance range widely from $10^{-8}$ to $10^{-5} \mathrm{~m}^{2} . \mathrm{K}^{\mathrm{W}} \mathrm{W}^{-1}$ for different cases from very good to poor contact, respectively, between the splat and the substrate $[4,8]$. Our overall objective in applying modeling to this problem is to examine the effect individually of various factors related to substrate conditions, or droplet conditions on the splat morphology. Previously, 
we have used three dimensional numerical models to study the splat spreading process and the phenomena of substrate melting when impacted by molten splats. In this case, we assumed that contact between the splat and substrate was perfect, i.e the thermal contact resistance is very low $[9,10]$. In this study, we numerically study the complex interaction between a molten metallic droplet (nickel) and solid substrate (stainless steel). Computational fluid dynamics (CFD) with the volume of fluid method (VOF) is used to stimulate the effect of thermal contact resistance at specific contact angle on the splat spreading behaviour. The models are validated against our own experimental observations regarding the fragmentation and splashing mechanism of the splat $[10,11]$.

Numerical model

In this work, the impact of a nickel droplet onto a smooth stainless steel substrate was modelled using a free surface model in which the fluids (droplet and air) were separated by a distinct resolvable interface. The surface tension at the droplet - air interface was modeled as a volume force based on the Continuum Surface Force model (CSF) of Brackbill et al. [12]. The following assumptions were made to model the present problem mathematically.

- $\quad$ The spherical droplet impact was normal on a smooth surface;

- The liquid flow was laminar;

- The velocity of the solid phase was zero;

- Tangential stresses at the free surface was neglected;

- No slip and no penetration boundary conditions were applied at the substrate surface;

- The effect of ambient during droplet impact was negligible;

- The viscous dissipation was neglected;

- Thermal contact resistance and surface tension were assumed to be constant in time;

- Density and viscosity are considered to vary from liquid phase to solid phase but are constant in each phase.

With the above assumptions, the equation of mass, momentum, and energy conservation for the binary solid-liquid phase change system of a droplet are presented as below [12-14].

Mass conservation

$$
\nabla \cdot u=0
$$

Momentum conservation

$$
\rho \frac{\partial u}{\partial t}+\rho \nabla \cdot(u u)=-\nabla p+\nabla \cdot\left(\mu \frac{\rho}{\rho_{l}} \nabla u\right)+\rho B
$$

Energy conservation

$$
\frac{\partial T}{\partial t}+\nabla \cdot(u T)=\alpha \nabla \cdot(\nabla T)-\frac{L}{C_{p l}} \frac{\partial f_{l}}{\partial t}+\frac{C_{p l}-C_{p s}}{C_{p l}} \frac{\partial}{\partial t}\left(f_{s} T\right)
$$


The relationship between droplet temperature and liquid mass fraction is:

$$
f_{l}=\frac{C_{p l}}{L}\left(T-T_{m}\right)
$$

The liquid and solid mass fractions are constrained to sum to unity, that is:

$$
f_{l}+f_{s}=1
$$

The locations of contact lines, the interface between the droplet and air fluids, and the shape and trajectory of the droplet were tracked using the "volume of fluid" (VOF) method [14]. The volume of fluid of the droplet (F) specifies the fraction of the volume of each computational cell in the grid occupied by the droplet and is defined to be unity for all the points inside the droplet and zero elsewhere. A value of $\mathrm{F}$ between 0 and 1 represents the interface between the droplet and air.

$$
\frac{\partial F}{\partial t}+\nabla \cdot(u F)=0
$$

The surface tension force per unit interface area is given by CSF model [12].

$$
F_{\beta \gamma}=\sigma \kappa_{\beta \gamma} n_{\beta \gamma} \delta_{\beta \gamma}+\left(\rho-\rho_{\gamma}\right) g
$$

The stress boundary condition at the free surface is:

$$
p_{\beta}-p_{\gamma}=-\sigma \kappa_{\beta \gamma}
$$

Heat transfer between the droplet and substrate was assumed to be due to heat conduction alone. A thermal contact resistance $\left(\mathrm{R}_{\mathrm{c}}\right)$ was used to account for the discontinuity at the interface between the droplet and the substrate:

$$
\frac{1}{R_{c}}\left(T-T_{s u b}\right)=k_{s u b} \frac{\partial T}{\partial x}
$$

The heat conduction through the substrate is written as:

$$
\frac{\partial T_{\text {sub }}}{\partial t}=\alpha_{\text {sub }} \nabla^{2} T_{\text {sub }}
$$

The governing equations are represented discretely in unstaggered, co-located grids and solved by an algebraic multi-grid solver for each control volume. The general solution algorithm for each time step is summarized in our previous papers $[9,10]$. A free surface domain for the droplet was made with the dimensions of 10r $\times 10 \mathrm{r} \times 2.1 \mathrm{r}$, where $\mathrm{r}$ is the initial radius of droplet. The depth of the substrate was chosen to ensure that the bottom substrate temperature is constant at $25^{\circ} \mathrm{C}$. Heat transfer with the phase change induced by inter-phase heat transfer inside the flow was incorporated into the program using a multiphase Eulerian-Eulerian fixed-grid model. The latent heat of fusion was indirectly obtained as the difference between static enthalpies of the two phases at the selected melting point and reference pressure of $1 \mathrm{~atm}$. Only a quarter of the domain was modeled to reduce processing time. The mesh size of 20 cells per initial droplet was applied for both droplet and substrate domain. Different values of thermal contact resistance and contact angle were examined. The contact angle is imposed in the code within the surface tension model in the solver. The contact angle is then used to adjust the surface normal in cells near the wall, and thus adjust the curvature and interface shape near the wall. This curvature is then used to adjust the body force term in the surface tension calculation. 
In order to validating the model, the nickel droplet was plasma sprayed onto stainless steel substrate using a dc plasma torch (PTF4 type) with a $6 \mathrm{~mm}$ diameter internal diameter nozzle and running with a mixture of hydrogen and argon. The arc current was set at 650A. The spray distance was $110 \mathrm{~m}$. The deformation process of the droplet was experimentally followed in time using a high speed camera (4,000 images/s). The initial velocity and temperature of the droplet were measured using the DPV-2000 system Tecnar, Canada) and were used as initial conditions for the numerical setup. The thermal contact resistance used in the model was adjusted empirically to give the best agreement of the splat shape between the modeling and empirical observations. Due to very high velocity at impact, Weber number $\left(\mathrm{We}=\rho v^{2} \mathrm{D} / \sigma=2,517\right)$ is large and thus surface tension and contact angle effects will initially negligible. Thus, the value static angle is reasonable can be used instead of dynamic contact angle.

Table 1: Physical properties of droplet and substrate.

\begin{tabular}{lcc}
\hline Physical properties & Nickel & Stainless steel \\
\hline Initial diameter $(\mathrm{D}, \mu \mathrm{m})$ & 20 & \\
Impact temperature $\left(\mathrm{T},{ }^{\circ} \mathrm{K}\right)$ & 2,427 & 25 \\
Impact velocity $(\mathrm{v}, \mathrm{m} / \mathrm{s})$ & 120 & \\
Melting point temperature $\left(\mathrm{T}_{\mathrm{m}},{ }^{\circ} \mathrm{K}\right)$ & 1,453 & \\
Surface tension $(\sigma, \mathrm{N} / \mathrm{m})$ & 1.78 & \\
Viscosity $(\mu, \mathrm{kg} / \mathrm{m} . \mathrm{s})$ & $3.3 \times 10^{-3}$ & \\
Latent heat of fusion $(\mathrm{L}, \mathrm{J} / \mathrm{kg})$ & $2.9 \times 10^{5}$ & \\
Thermal conductivity of liquid $\left(\mathrm{k}_{\mathrm{l}}, \mathrm{W} / \mathrm{m} . \mathrm{K}\right)$ & 43 & \\
Thermal conductivity of solid $\left(\mathrm{k}_{\mathrm{s}}, \mathrm{W} / \mathrm{m} . \mathrm{K}\right)$ & 80 & 28 \\
Specific heat capacity of liquid $\left(\mathrm{C}_{\mathrm{pl}}, \mathrm{J} / \mathrm{kg} . \mathrm{K}\right)$ & 620 & \\
Specific heat capacity of solid $(\mathrm{Cps}, \mathrm{J} / \mathrm{kg} . \mathrm{K})$ & 595 & 690 \\
Density of liquid $\left(\rho_{\mathrm{l}}, \mathrm{kg} / \mathrm{m}^{3}\right)$ & 7,780 & \\
Density of solid $\left(\rho_{\mathrm{s}}, \mathrm{kg} / \mathrm{m}^{3}\right)$ & 8,450 & 7,854 \\
\hline
\end{tabular}

\section{Results and discussion}

\subsection{Splat morphology as a function of thermal contact resistance}

The impact of a molten nickel droplet on a stainless steel substrate was examined at different values of thermal contact resistance which was fine-tuned in the range of reported values from $5 \times 10^{-8}$ to $2 \times 10^{-7} \mathrm{~m}^{2} . \mathrm{K}^{-W^{-1}}$ for different cases from good to poor contact, respectively, between the splat and the substrate [8]. Figure 1 shows the simulated sequence of a $20 \mu \mathrm{m}$ diameter nickel splat on a stainless steel substrate which was held at room temperature during the impact of the droplet. The contact angles were kept constant at $60^{\circ}$ in all cases. The time elapsed from the moment of impact is indicated next to each frame. Upon impact, the droplet flattened and spread out in radial direction to form a pancake splat with the maximum diameter of $48 \mu \mathrm{m}$ at $0.2 \mu \mathrm{s}$ approximately. Due to the 

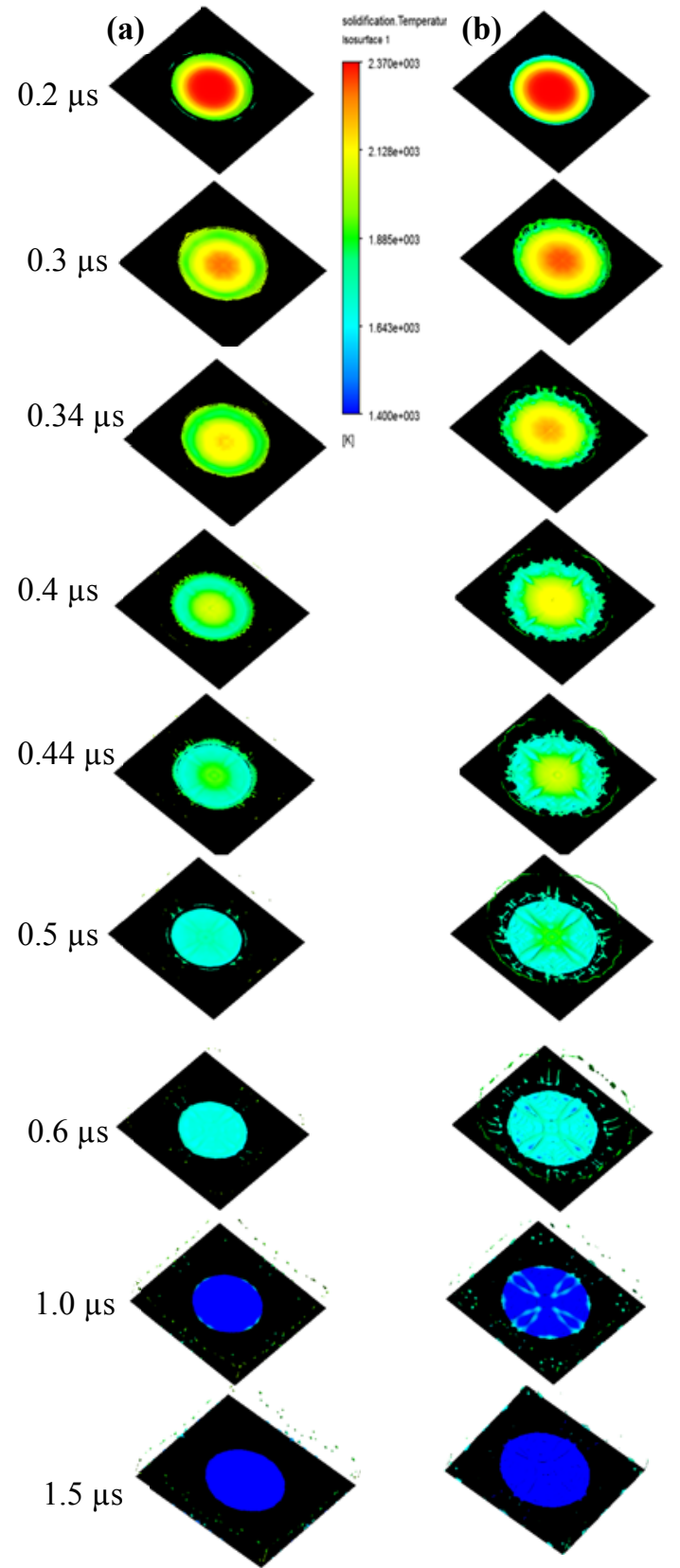

(c)
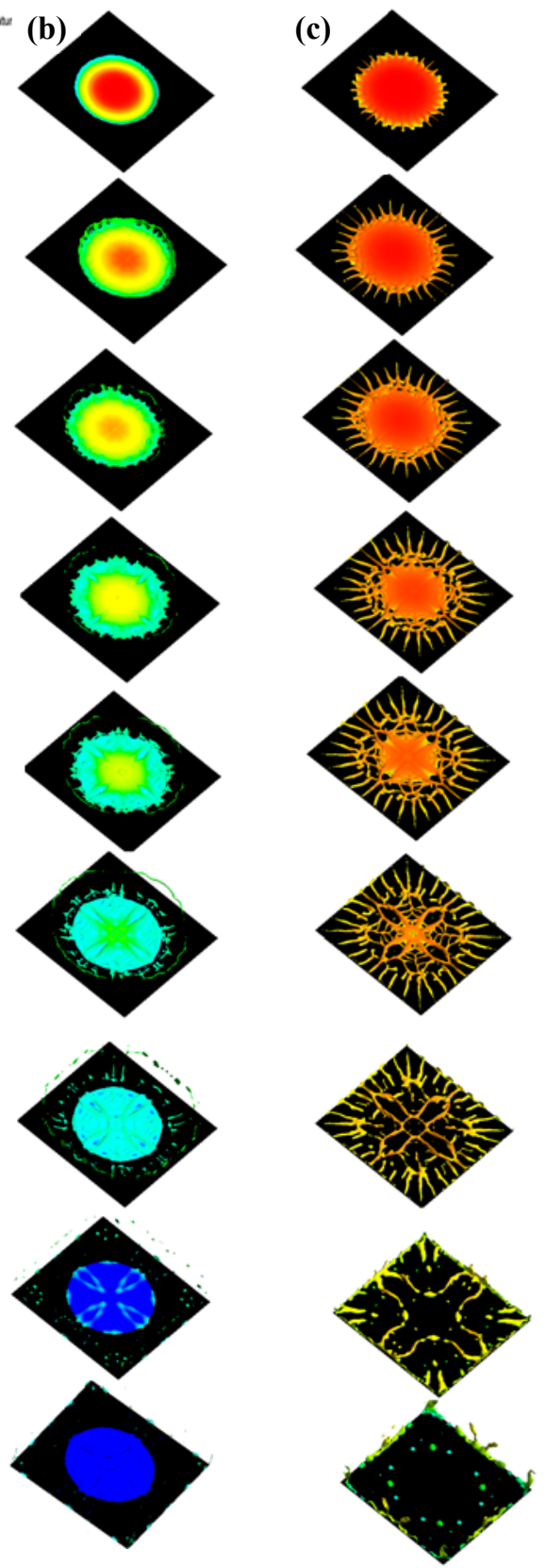

Figure 1: The sequence of the numerical simulation of a $20 \mu \mathrm{m}$ diameter nickel splat onto stainless steel substrate with $\mathrm{R}_{\mathrm{c}}$ of (a) $5 \times 10^{-8} \mathrm{~W}^{-1} \cdot \mathrm{m}^{2} . \mathrm{K}$; (b) $10^{-7} \mathrm{~W}^{-1} \cdot \mathrm{m}^{2} . \mathrm{K}$; and (c) $2 \times 10^{-7} \mathrm{~W}^{-1} \cdot \mathrm{m}^{2} . \mathrm{K}$ with the contact angle of $60^{\circ}$. 
high velocity of the droplet, the spreading in the early stage is dominated by inertial energy only. During this time, the droplet was totally in liquid form and thus solidification has made no effect on the spreading kinetics and the final splat shape. Thus, the spread factor is almost the same for all the case initially.

With the low thermal contact resistance as $5 \times 10^{-8} \mathrm{~W}^{-1} \cdot \mathrm{m}^{2} \cdot \mathrm{K}$, the splat was in good contact with the substrate. It was observed from the Figure 1(a) that the splat subsequently disintegrates at the splat edge. Then the disintegration moves towards the central part of the splat. The break-up process completes at a time of approximately $1 \mu \mathrm{s}$, leaving a central core remaining on the surface, surrounded by a circular ring of debris. Figure 1(b) displays the case with higher thermal contact resistance $\left(10^{-7} \mathrm{~W}^{-1} \cdot \mathrm{m}^{2} . \mathrm{K}\right)$ which represents for the case of poor contact between the splat and the substrate. It was observed that after spreading to a maximum extent as the case of lower thermal contact resistance (Figure 1(a)), the splat started breaking up at the edge. In addition, the surface of the central part of the splat is not as smooth as the case of lower thermal contact resistance. Due to higher thermal contact resistance, the splat had longer time in the fluid form. At this time, it was expected that flow resistance is induced by surface tension and viscosity of the droplet. Owing to surface tension, the edge of the splat retracts relative to the flow in the droplet, and fluid collects into a toroid rim bounding the splat. Thus, there is a competence between the flowing fluid in radial direction at the edge due to the kinetic and the retraction due to the surface tension. The area near the edge become unstable and results in the perturbation in this area, resulting in the break-up at the edge and the uneven of the splat surface as observing in Figure 1(b). If the contact between the splat and substrate is not good (in the case of higher of thermal contact resistance, $2 \times 10^{-7} \mathrm{~W}^{-1} \cdot \mathrm{m}^{2} . \mathrm{K}$ ), the perturbation then continues to move further towards the centre of the splat, resulting in significant fragmentation of the splat. This movement finally generates the complete break-up of the splat that leaves only tiny debris on the substrate surface as seen in Figure 1(c). Thus, the splashing mechanism in our model is attributed to the instability of rapidly decelerating interface and the subsequent formation of dominant surface tension as discussed further below.

\subsection{Splat morphology as a function of contact angle}

To further examine how the contact angle influences the droplet deformation process and thus splat morphology, in this part, the thermal contact resistance was kept at $10^{-7} \mathrm{~W}^{-1} \cdot \mathrm{m}^{2} . \mathrm{K}$ with the contact angle either at $60^{\circ}$ or $90^{\circ}$. Figure 2 shows images of droplet deformation obtained from the numerical model as well as the droplet velocity evolution (half cross section) during the spreading process. It was observed that the contact angles have little influence on early stages of droplet spread (up to $0.2 \mu \mathrm{s}$ ): the droplets spread to a maximum extent with the curling at the edge of the splat. Similar to the previous cases, the droplet spreading during the initial period was dominated by inertial force rather than viscous force. Therefore, the variations of either contact angle or thermal contact resistance have little influence on fluid flow at the initial stage. 


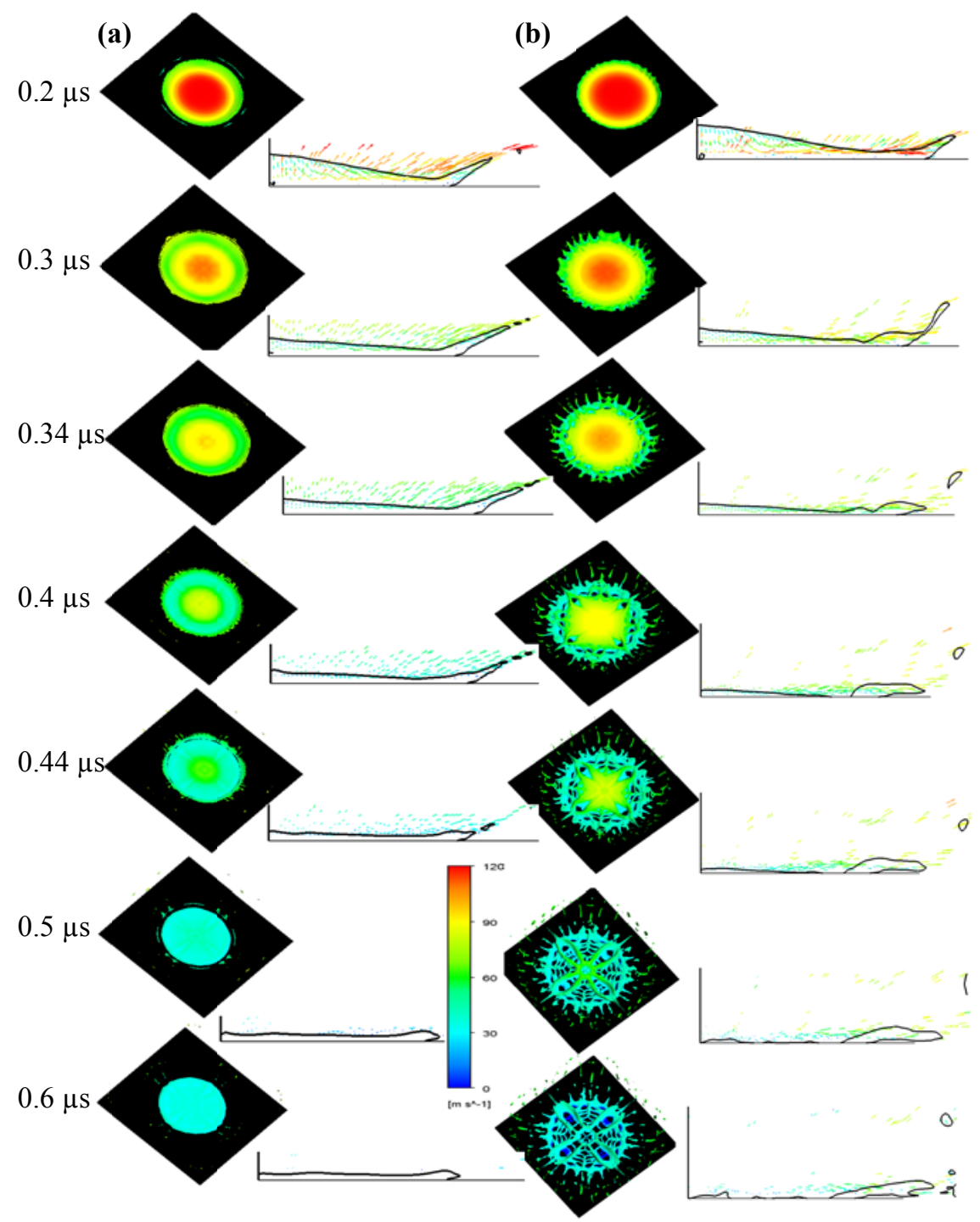

Figure 2: The sequence of the numerical simulation of a nickel splat onto stainless steel substrate with $\mathrm{R}_{\mathrm{c}}$ of $10^{-7} \mathrm{~W}^{-1} \cdot \mathrm{m}^{2} \cdot \mathrm{K}$ and contact angle of (a) $60^{\circ}$ and (b) $90^{\circ}$. The half cross section of the droplet represents for the velocity vector during spreading process. The vertical bar indicates values of the velocity vector of the droplet.

It was observed from the Figure 2(b) that at the maximum extent, the velocity of the splat at the edge in the case of the contact angle of $90^{\circ}$ is much higher that of contact angle of $60^{\circ}$. The splat at the edge has the tendency to move outwards quickly (as can be seen in velocity field in Figure 2(b)) while the surface tension 
retracts the splat inwards. In addition, poor contact with the substrate (as a result of the high contact angle) results in poor heat conduction from the splat to the substrate. The splat is in liquid state for longer time, and thus easily break-up. The splat again has an uneven surface and significant ruptures, but not completely break-up. This is the intermediate case between the case with thermal contact resistance of $10^{-7} \mathrm{~W}^{-1} \cdot \mathrm{m}^{2} \cdot \mathrm{K}$ and (c) $2 \times 10^{-7} \mathrm{~W}^{-1} \cdot \mathrm{m}^{2} \cdot \mathrm{K}$ (Figure $1(\mathrm{~b})$ and $1(c))$ with the smaller contact angle of $60^{\circ}$. These numerical results show the influence of contact angle on the splat morphology upon impact the wall at high velocity. As the contact angle is imposed in the code within the surface tension model in the solver. The contact angle is then used to adjust the surface normal in cells near the wall, and thus adjust the curvature and interface shape near the wall. This curvature is then used to adjust the body force term in the surface tension calculation.

It was also found that the splat curled up at the edge independently on thermal contact resistance and contact angle. Upon impact the substrate, the pressure at the contact point rapidly increased to $170 \mathrm{MPa}$ approximately (Figure 3(a)). The contact pressure subsequently decreases during the droplet spreading. However, the contact pressure at the periphery is significantly smaller than that at the central splat (Figure 3(b)). Thus, the impact pressure at the periphery of the splat is not enough to overcome the capillary pressure required to force liquid into the surface crevices where it would otherwise solidify and bond to the substrate. Without this bond, the splat edge could not be kept in place against the surface tension which pulls the splat edge inwards. The direction of the velocity vector in this model, as displayed in Figure 2, also indicated the tendency of curling up of the splat at its perimeter.
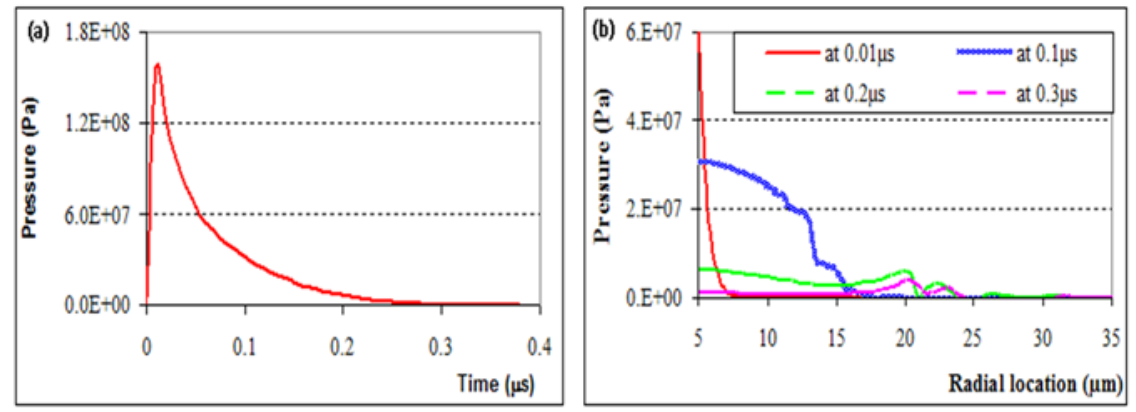

Figure 3: (a) Pressure distribution at the contact point, and (b) pressure distribution as a function of time and radial location.

To validate the numerical modelling, nickel droplet was plasma sprayed on stainless steel substrates. The droplet spreading process is captured using high speed camera. A polished substrate has been sprayed at room temperature represented for the case of the good contact between the splat and the substrate whereas modified substrate by hydrothermal treatment indicated a poor contact between the splat and the substrate as reported in our previous studies $[10,11$, 
$15,16]$. It is observed that for the case of good contact between the droplet and the substrate, the droplet spreads to a maximum extent and then ruptures starting from the splat edge and then moves towards the central part of the splat, as shown in Figure 4(a). The final splat shape is the central surrounding with a ring of debris as in SEM image in Figure 4(b). For the case of poor contact, the droplet spreads to maximum extent as well, then keep moving outwards and finally splashing off from the surface (Figure 4(c)), leaving a very thin film on the surface as can be seen in Figure 4(d). The SEM image of the splat from the empirical observation (Figure 4(b) and 4(d)) had a similar morphology to the predicted splat from the modeling work shown in Figure 2(a) and 2(c). Thus, using contact angle of $60^{\circ}$ or $90^{\circ}$ and thermal contact resistance $5 \times 10^{-8} \mathrm{~W}^{-1} \cdot \mathrm{m}^{2} \cdot \mathrm{K}$ or $2 \times 10^{-7} \mathrm{~W}^{-1} \cdot \mathrm{m}^{2} . \mathrm{K}$ give predictions that were in close agreement with experimental observations. Therefore, the change in splat morphology is related to the contact angle (which changes the surface tension force and then adjust the body force term in the surface tension calculation) and thermal contact resistance.

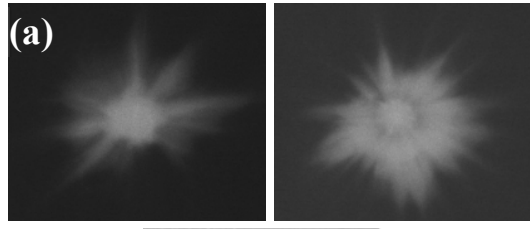

(b) SEM image

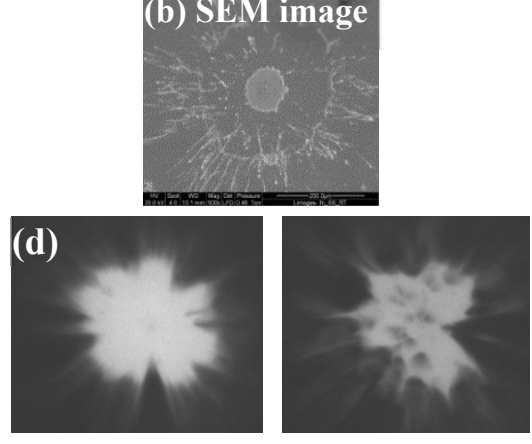

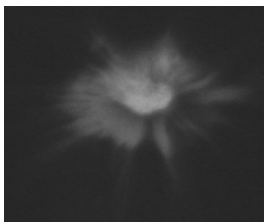

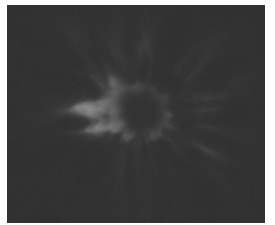

(c) SEM image

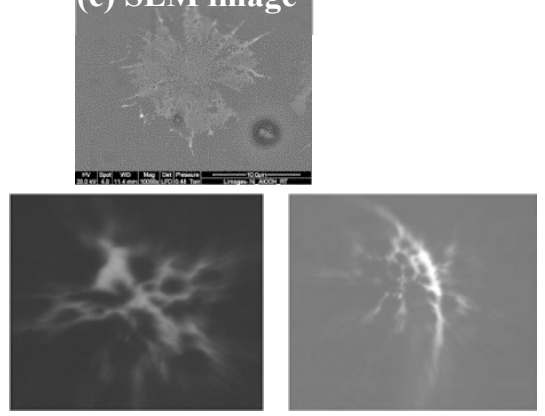

Figure 4: The sequence of the empirical observations of a nickel splat onto stainless steel substrate with (a)-(b) good contact, and (c)-(d) poor contact between the splat and the substrate.

It was also expected that the break-up as observed may be the result of Rayleigh-Taylor instability, which occurs when a light fluid is accelerated into a heavy fluid. The role of Rayleigh-Taylor instabilities in splashing has been proposed by Allen [17]. However, the detailed mechanism involved has not been studied fully yet. In our model, the light fluid is air (density $1.2 \mathrm{~kg} . \mathrm{m} \mathrm{-3}$ ) and the heavy fluid is liquid droplet (density 7,780 kg.m -3). We propose that upon the impact of the droplet, air is trapped at the splat-substrate interface. High 
velocity of the liquid droplet, which causes a pressure build-up at the dropletsubstrate interface, induces a good contact of the splat with the substrate. As the droplet spreads on the substrate, the pressure and the overall fluid velocity rapidly decrease. At the maximum extent, surface tension is more dominant than the inertial force. Due to the surface tension and the variation of the velocity field of the fluid flow along the contact line between the droplet, air and substrate at the periphery, the leading edge become unstable and result in the perturbation at this location. The perturbation breaks up the stability of the interface between the liquid splat and air. The heavier splat moves down under the effect of gravity, whereas the lighter air tends to accelerate through the splat. The Rayleigh-Taylor instabilities initially provoke the detachment of liquid splat at the periphery. The perturbation then continues to move further towards the centre of the splat, resulting in a significant fragmentation of the splat. This movement generates the satellite droplets that forms a ring of debris surrounded the central core of the splat. At a higher extent, the disintegration continues to the central core of the splat, resulting a complete splashing to form small fragmented droplets on the surface. Thus, the splashing mechanism in our model is attributed to the instability of a rapidly decelerating interface and the subsequent formation of dominant surface tension due to the contact angle.

\section{Conclusion}

The influence of thermal contact resistance and contact angle on the impact dynamic of a micrometre-sized nickel droplet at high velocity onto a stainless steel is studied using numerical modelling basing on computational fluid dynamics method. The model is validated against our own empirical observations. The principle findings were summarised as below:

- $\quad$ The droplet spreading and thus the splat morphology strongly depend on thermal contact resistance and the contact angle between the droplet and the substrate. High values of thermal contact resistance or contact angles which represents for the case of bad contact between the droplet and the substrate can induce droplet breakup and even totally splashing. This break-up is due to the instability of a rapidly decelerating interface and the subsequent formation of dominant surface tension related to the thermal contact resistance and contact angle respectively. The splashing can be avoided with lower values of both thermal contact resistance and contact angle which indirectly means good contact between the droplet and the substrate at the time of droplet impact.

- Comparison of computer generated images with the high speed cameracaptured photographs of the droplet showed that the splat morphology as well as the splashing mechanism of the droplet during deformation was in good agreement with the empirical observations. Thus, the numerical results can be used to provide new insights into the interpretation of empirical results, enhance the physical understanding of the fluid mechanism that leads to splashing, as well as predict and improve the splat spreading process and thus the quality of coatings at a larger scale. 


\section{References}

[1] Fauchais, P., et al., Knowledge concerning splat formation: an invited review. J. Therm. Spray. Techn., 2004. 13(3): pp. 337-360.

[2] Chandra, S. and P. Fauchais, Formation of solid splats during thermal spray deposition. J. Therm. Spray. Techn., 2009. 18(2): pp. 148-180.

[3] Pasandideh-Fard, M., S. Chandra, and J. Mostaghimi, A three-dimensional model of droplet impact and solidification. Int. J. Heat Mass Tran., 2002. 45: pp. 2229-2242.

[4] Pasandideh-Fard, M., et al., Splat shapes in a thermal spray coating process: simulations and experiments. J. Therm. Spray. Techn., 2001. 11: pp. 206-217.

[5] Harlow, F.H. and J.P. Shannon, The splash of a liquid drop J. Appl. Phys., 1967. 38(10): pp. 3855-3866.

[6] McDonald, A., et al., Modeling fragmentation of plasma-sprayed particles impacting on a solid surface at room temperature. Comptes Rendus Mecanique, 2007. 335: pp. 351-356.

[7] Heichal, Y. and S. Chandra, Predicting thermal contact resistance between molten metal droplets and a solid surface. J. Heat Transfer 2005. 127: pp. 1269-1275.

[8] McDonald, A., C. Moreau, and S. Chandra, Thermal contact resistance between plasma-sprayed particles and flat surfaces. Int. J. Heat Mass Transfer, 2007. 50: pp. 1737-1749.

[9] Tran, A.T.T., et al., Evidence of substrate melting of $\mathrm{NiCr}$ particles on stainless steel substrate by experimental observation and simulations Plasma Chem. Plasma P., 2009. 29(6): pp. 475-495.

[10] Tran, A.T.T. and M.M. Hyland, The role of substrate surface chemistry on splat formation during plasma spray deposition by experiments and simulations. J. Therm. Spray. Techn., 2010. 19(1-2): pp. 11-23.

[11] Tran, A.T.T., et al., Inhibition of molten droplet deposition by substrate surface hydroxides. Surf. Coat. Technol., 2011. 206(6): pp. 1283-1292.

[12] Brackbill, J.U., D.B. Kothe, and C. Zemach, A continuum method for modelling surface tension. J. Compu. Phys., 1992. 100(2): pp. 335-354.

[13] Park, H., et al., Single drop impaction on a solid surface. AIChE Journal, 2003. 49(10): pp. 2461-2471.

[14] Prakash, C. and V. Voller, On the numerical solution of continuum mixture model equations describing binary solid-liquid phase change. Numer. Heat Tr., B- Fund., 1989. 15(2): pp. 171-189.

[15] Tran, A.T.T., et al., Effects of surface chemistry on splat formation during plasma spraying. J. Therm. Spray. Techn., 2008. 17(5-6): pp. 637-645.

[16] Tran, A.T.T., et al., Influence of substrate surface conditions on the deposition and spreading of molten droplets Thin Solid Films, 2011. 519: pp. 2445-2456.

[17] Allen, R.F., The role of surface tension in splashing. Journal of Colloid and Interface Science, 1975. 51(2): pp. 350-351. 